\title{
OsNBL3, a mitochondria-localized pentatricopeptide repeat protein, is involved in splicing nad5 intron 4 and responding to biotic and abiotic stresses
}

\author{
Tiancheng Qiu ${ }^{1}$, Xiaosheng Zhao ${ }^{1}$, Huijing Feng ${ }^{1}$, Jun Yang ${ }^{1}$, You-Liang Peng ${ }^{1}$, and \\ Wensheng Zhao ${ }^{2}$ \\ ${ }^{1}$ China Agricultural University, College of Plant Protection Yuanmingyuan West road No.2 \\ Beijing, CN 100193 \\ ${ }^{2}$ China Agricultural University
}

October 20, 2020

\begin{abstract}
Lesion mimic mutants are used to elucidate mechanisms controlling plant responses to pathogen attacks and environmental stresses. Here, a new rice (Oryza sativa) lesion mimic mutant, natural blight leaf 3 (nbl3), was identified from T-DNA insertion lines. The causative gene, OsNBL3, encodes a mitochondria-localized pentatricopeptide repeat (PPR) protein. The nbl3 mutant exhibited spontaneous cell death and $\mathrm{H}_{2} \mathrm{O}_{2}$ accumulation as evidenced by Trypan blue, 3,3'-diaminobenzidine and tetranitroblue tetrazolium chloride analyses. Additionally, nbl3 displayed enhanced resistance to the fungal and bacterial pathogens Magnaporthe oryzae and Xanthomonas oryzae pv. oryzae. This resistance was consistent with the upregulation of several defence-related genes; thus defence responses were induced in nbl3. RNA interference lines of OsNBL3 exhibited enhanced disease resistance similar to that of $n b l 3$, while the disease resistance of overexpression lines did not differ from that of the wild type. In addition, nbl3 displayed improved tolerance to salt treatment, accompanied by upregulation of several saltassociated marker genes. Disruption of $O s N B L 3$ leads to destruction of mitochondria and elevated use of alternative respiratory pathways. OsNBL3 was found to mainly participate in the splicing of mitochondrial gene nad5 intron 4 . Overall, the results demonstrated that a PPR protein was involved in disease resistance and salt tolerance in rice.
\end{abstract}

\section{KEYWORDS}

disease resistance, salt tolerance, pentatricopeptide repeat (PPR) protein, mitochondria, RNA splicing, nad5, lesion mimic mutant, Oryza sativa, OsNBL3

\section{INTRODUCTION}

Programmed cell death (PCD) plays important roles in the embryonic, juvenile and adult phases of plant development. In addition, a type of PCD is often observed during plant response to pathogen attack; this is termed the 'hypersensitive response' (HR) (Jones \& Dangl, 1996). HR is a defence mechanism employed by plants to protect themselves from being disturbed by external conditions. The occurrence of HR in plants is usually accompanied by several typical characteristics, including generation of reactive oxygen species (ROS), expression of pathogenesis-related (PR) proteins, accumulation of calluses, and thickening of cell walls. These changes can help plants resist a variety of pathogens (Greenberg, Guo, Klessig, \& Ausubel, 1994).

Lesion mimic mutants (LMMs), also named spotted leaf ( $s p l$ ) mutants, spontaneously develop localized cell death lesions resembling those caused by HR in the absence of pathogen infection, abiotic stress or 
mechanical damage. In many cases, LMMs exhibit significantly enhanced disease resistance (Xu, Zhang, Liu, Ye, \& Wu, 2014). Therefore, LMMs are deemed as suitable materials and have frequently been used for elucidating the mechanisms underlying plant responses to pathogen attacks and environmental stresses. To date, dozens of genes have been characterized from LMMs that encode various types of proteins, including porphyrin (Ishikawa, Okamoto, Iwasaki, \& Asahi, 2001; Undan et al., 2012), transcription factors (Li, Brader, \& Palva, 2004; Yamanouchi, Yano, Lin, Ashikari, \& Yamada, 2002), oxidoreductases (Tanaka, Hirashima, Satoh, \& Tanaka, 2003; Yang, Wardzala, Johal, \& Gray, 2004), protein kinases (Liang et al., 2003; S. H. Wang et al., 2015), ubiquitinations (Zeng et al., 2004), membrane-associated proteins (Lorrain et al., 2004; Noutoshi et al., 2006), zinc finger proteins (Wang, Pei, Tian, \& He, 2005), ion channel proteins (Mosher et al., 2010; Rostoks et al., 2006), clathrin-associated adaptor proteins (Qiao et al., 2010), nucleotide binding site-leucine-rich repeat (NBS-LRR) type proteins (Tang et al., 2011), mRNA splicing factors (Chen et al., 2012), UDP- $N$-acetylglucosamine pyrophosphorylase (Z. Wang et al., 2015), AAA-type ATPase (Fekih et al., 2015), eEF1A-like protein (S. Wang et al., 2017), and glycine-rich domain proteins (Zhao et al., 2020). The large range of protein types found to contribute to the characteristics of LMMs suggests that the molecular mechanisms regulating defence responses in plants are very complicated.

Mitochondria function as energy processing factories and provide energy for life activities in eukaryotes. The respiratory electron transport chain (ETC) is composed of respiratory enzyme complexes that are embedded or anchored in the inner membranes of mitochondria. These protein complexes include the nicotinamide adenine dinucleotide (NADH)-ubiquinone oxidoreductase system (complex I), succinate-ubiquinone reductase system (complex II), cytochrome b precursor (complex III), cytochrome c oxidase system (complex IV) and ATP synthase system (complex V). The proteins comprising these complexes, excluding complex II, are encoded by the mitochondrial genome. These complexes can transfer electrons to oxygen and supply the energy metabolism of cells through redox reactions. If oxygen cannot be fully reduced, ROS will be produced. When the function of genes involved in the respiratory ETC is abnormal, the loss of ETC function leads to electron leakage. It has been reported that the ROS produced by mitochondria are superoxide and $\mathrm{H}_{2} \mathrm{O}_{2}$ (Huang, Van Aken, Schwarzlander, Belt, \& Millar, 2016). In mammals and plants, superoxide is formed by the reduction of $\mathrm{O}_{2}$ by electrons that have accumulated in respiratory complexes I and III in the ETC (Blokhina \& Fagerstedt, 2010). However, plant mitochondria can bypass the oxidative phosphorylation pathway and transport protons without producing ATP, by depending on alternative NAD $(\mathrm{P}) \mathrm{H}$ dehydrogenases (NDs) and alternative oxidases (AOXs) to reduce the superoxide produced by the mitochondrial ETC (Vanlerberghe, 2013). Plant mitochondrial ROS (mtROS) are involved in redox signalling (Suzuki, Koussevitzky, Mittler, \& Miller, 2012), PCD (Van Aken \& Van Breusegem, 2015; J. Wu et al., 2015), plant hormone signalling (Colombatti, Gonzalez, \& Welchen, 2014; Poor et al., 2019), retrograde signals and defence against pathogens (Colombatti et al., 2014; Huang et al., 2016; Lyu et al., 2019).

Although plant mitochondrial genomes can encode genes, most of these genes are regulated by proteins encoded by nuclear genes (Schmitzlinneweber \& Small, 2008). Among the proteins involved in mitochondrial gene expression regulation, pentatricopeptide repeat (PPR) proteins play a unique role. The PPR protein family is one of the largest protein families in higher plants. Approximately 400-700 PPR genes are present in sequenced angiosperm genomes (Cheng et al., 2016). PPR proteins can be divided into P and PLS subfamilies according to the different PPR motifs. The P-subfamily proteins only consist of canonical PPR motifs that contain 35 amino acids, while the PLS-subfamily proteins also contain short motifs ( $\mathrm{S} ; 31$ amino acids), long motifs (L; 35-36 amino acids) and E, E+ or DYW domains at the C-terminus (Schmitzlinneweber \& Small, 2008). In rice, a total of 491 PPR proteins have been predicted, including 246 P-subfamily and 245 PLS-subfamily members. Moreover, 90 E domains and 131 DYW domains have been found in the PLS subfamily (Chen, Zou, Hu, \& Ding, 2018). P-subfamily PPR proteins are mainly involved in mitochondrial RNA splicing, stabilization and translation, while PLS-subfamily proteins mainly play a role in the C-U editing of mitochondrial RNA (Barkan \& Small, 2014).

Most of the PPR proteins located in mitochondria are involved in modifying the gene expression of subunits of the mitochondrial ETC complexes. Mutations in PPR genes lead to dysfunction of the mitochondrial ETC, which can lead to seed and embryo development defects, growth retardation, pollen abortion, abiotic 
stress and ABA sensitivity in plants (Barkan \& Small, 2014). In Arabidopsis, the P-subfamily proteins MTL1 (Haili et al., 2016), slow growth 3 (Hsieh et al., 2015) and OTP43 (De Longevialle et al., 2007) have been shown to be localized to the mitochondria and are involved in thecis - or trans -splicing of the introns of genes encoding subunits of complex I. Recently, it was reported that a rice P-subfamily PPR protein, FLO10, is required for the trans -splicing of the mitochondrial nad1 intron 1 (Wu et al., 2019). Another P-subfamily PPR protein, RL1 of rice, was specifically involved in the splicing of the mitochondrial nad4intron 1 (Wu, Zhao, Zhang, Guo, \& Liu, 2020). Several mitochondria-localized P-type PPR proteins have been reported to be involved in the RNA stabilization of genes encoding subunits of complex I. These P-type PPR proteins include Arabidopsis AtMTFS1 (Haili et al., 2013), AtMTFS2 (C. D. Wang et al., 2017), PPR19 (Lee et al., 2017) and maize PPR78 (Zhang, Suzuki, Sun, \& Tan, 2017). The mitochondria-localized PLS-subfamily PPR proteins, including rice OGR1 (Sungryul et al., 2009), MPR25 (Toda, Fujii, Noguchi, Kazama, \& Toriyama, 2012), Arabidopsis SLO2 (Zhu et al., 2012), SLO4 (Weissenberger, Soll, \& Carrie, 2017), MEF8 (Diaz, Bentolila, Hayes, Hanson, \& Mulligan, 2017), maize DEK36 (G. Wang et al., 2017) and DEK39 (Li et al., 2018) are mainly involved in the C-U editing of genes encoding subunits of complexes I and III. Although these studies shed some light on the roles of PPR proteins in plant growth and development by regulating mitochondrial RNA metabolism, the functions of most PPR proteins remain unclear.

In this study, it was found that OsNBL3 encodes a mitochondria-localized P-subfamily PPR protein. The mutation of $O s N B L 3$ leads to a spontaneous lesion mimic phenotype accompanied by enhanced disease resistance and salt tolerance, and premature leaf senescence. RNA interference (RNAi) lines of OsNBL3 exhibited enhanced disease resistance similar to that of the nbl3 mutant, while the disease resistance of overexpression lines did not differ from that of the wild type. OsNBL3 mainly participates in the cis -splicing of nad5 intron 4, and thus contributes to the precise assembly and construction of mitochondria.

\section{MATERIALS AND METHODS}

\subsection{Plant materials and growth conditions}

The nbl3 mutant was identified by screening a T-DNA insertion population of the rice cultivar Aichiasahi (Oryza sativa 'Geng' [japonica]). The nbl3 mutant and wild-type plants were grown in the experimental field at China Agricultural University in Beijing, China, or in growth rooms maintained at $28^{\circ} \mathrm{C}$ during the day, with a 12-h light/12-h dark photoperiod and 70\% humidity.

\subsection{Pathogen inoculations and salt treatments}

Rice seedlings were inoculated with the Magnaporthe oryzaeisolate H535 using the punch inoculation method (Fang et al., 2018). In brief, the detached leaves of five-leaf-stage rice seedlings were wound-inoculated with $10 \mu \mathrm{L}$ of spore suspension $\left(2 \times 10^{5}\right.$ spores $\left./ \mathrm{mL}\right)$ supplemented with $0.025 \%$ Tween 20 . Moreover, intact leaves were spray-inoculated with H535 spore solution $\left(1 \times 10^{6}\right.$ spores $\left./ \mathrm{mL}\right)$ containing $0.025 \%$ Tween 20 . The inoculated leaves were then transferred to a chamber at $28^{\circ} \mathrm{C}$ under $100 \%$ humidity and a 12 -h dark $/ 12$-h light cycle. The length of the resulting lesions was measured at 96 hours post inoculation (hpi). To evaluate rice bacterial blight disease resistance, the wild type and nbl3 mutant were inoculated with the Xanthomonas oryzae pv. oryzae (Xoo ) strain PXO99 using the leaf-clipping method at 60 days after sowing (DAS). The overnight liquid culture of the bacterium was collected and adjusted to optical density (OD) $=0.8$ using deionized water. The distal tip (approximately $3 \mathrm{~cm}$ ) of the flag leaves was removed using scissors and then dipped into the bacterial suspension. Five individual plants and three tillers per plant were inoculated with PXO99, and these plants were grown in a glasshouse. The lesion length was measured at 14 days post inoculation (dpi).

For the salt treatments, seeds of the $n b l 3$ and wild-type plants were treated with sodium hypochlorite solution (1\%) to accelerate germination. Seedlings were planted on agar plates containing $0 \mathrm{mM}$ or $100 \mathrm{mM} \mathrm{NaCl}$ in a greenhouse $\left(28^{\circ} \mathrm{C}\right.$ and photoperiod of 12 -h light/12-h dark $)$. The seedlings were photographed and the root lengths were measured at 2, 3, 4 and $5 \mathrm{~d}$. In addition, 10-day-old nbl3 and wild-type seedlings grown in normal conditions $\left(28^{\circ} \mathrm{C}\right.$ and photoperiod of 12 -h light/12-h dark) were continuously irrigated with $200 \mathrm{mM}$ $\mathrm{NaCl}$, while unirrigated plants were used as the control. The whole plants were photographed after forty 
days of irrigation. During the irrigation with $\mathrm{NaCl}$, wild-type leaves were sampled at different time points to conduct expression analysis of $O s N B L 3$ by real-time quantitative polymerase chain reaction (RT-qPCR).

\subsection{SiteFinding thermal asymmetric interlaced (TAIL)-PCR for OsNBL3 cloning}

SiteFinding TAIL-PCR procedures were used to isolate the sequences flanking the T-DNA using previously described methods (Tan et al., 2005). The products of tertiary SiteFinding TAIL-PCR were sequenced and used to search against the rice genome database (http://rice.plantbiology.msu.edu/) to obtain the T-DNA insertion site. Specific primers, L3, R3, JD-F and JD-R, were used to reconfirm the T-DNA insertion site. The sequences of all of the primers used in the study are listed in Supporting Information Table S1.

\subsection{Transmission electron microscopy}

Leaf sections of the $n b l 3$ and wild type were sampled from plants at $60 \mathrm{DAS}$, and immersed in $2.5 \%$ glutaraldehyde in a phosphate buffer at room temperature for $48 \mathrm{~h}$ and then maintained at $4^{\circ} \mathrm{C}$ overnight. Subsequently, the samples were washed and incubated in $1 \% \mathrm{OsO}_{4}$ at $4^{\circ} \mathrm{C}$ for $12 \mathrm{~h}$. After dehydration in a gradient ethanol series, the samples were embedded in Spurr's resin prior to ultrathin sectioning. Sections were stained with uranyl acetate and examined with a Hitachi-H7500 transmission electron microscope.

\subsection{Histochemical assays}

The second leaves of the nbl3 and wild type were used in histochemical assays at 60 DAS. A lactic acidphenol-trypan blue solution was used to evaluate cell death, and tetranitroblue tetrazolium chloride (NBT) solution and 3,3'-diaminobenzidine (DAB) solution were used to evaluate $\mathrm{H}_{2} \mathrm{O}_{2}$ accumulation. Staining was performed using previously described methods (S. Wang et al., 2017). Briefly, leaves of the $n b l 3$ and wild-type plants were immersed in Trypan blue solution (0.25\% Trypan blue, $25 \%$ lactic acid, $23 \%$ watersaturated phenol and $25 \%$ glycerol) in a boiling water bath for $20 \mathrm{~min}$, cooled all night and supplemented with chloral hydrate $(0.25 \%)$ for $30 \mathrm{~h}$. Leaves were also immersed in DAB solution $(1 \mathrm{mg} / \mathrm{mL}$ DAB and 10 $\left.\mathrm{mM} \mathrm{Na} 2 \mathrm{HPO}_{4} ; \mathrm{pH} 3.8\right)$ or NBT solution $\left(0.5 \mathrm{mg} / \mathrm{mL} \mathrm{NBT}\right.$ and $\left.10 \mathrm{mM} \mathrm{K} \mathrm{HPO}_{4} ; \mathrm{pH} 7.8\right)$ in the dark for $16 \mathrm{~h}$ at room temperature. The staining leaves were transferred into $95 \%$ ethanol to decolourize.

\subsection{Subcellular localization}

To perform transient expression analysis in rice protoplasts andNicotiana benthamiana leaf epidermal cells, the full-length coding sequence of $O s N B L 3$ without the stop codon and with a total length of $300 \mathrm{bp}$ starting from ATG was amplified using the primer set OsNBL3-GFP-F/R or 3N-GFP-F/R, respectively. The PCR product was fused in-frame with green fluorescent protein (GFP) in the pCAMBIA1301 plasmid, to generate 35S:OsNBL3-GFP or 35S:3N-GFP fusion constructs, respectively. The expression vector was introduced into rice protoplasts following previous described methods (Zhao et al., 2020), and the transfected protoplasts were incubated at $28^{\circ} \mathrm{C}$ in the dark. The fluorescent GFP signal was examined and photographed using a laser confocal scanning microscope (Leica TCS SP8) after $16 \mathrm{~h}$. The mitochondria dye Mito-Tracker Red (Invitrogen) was incubated at $37^{\circ} \mathrm{C}$ in a suspension containing the rice protoplasts 15 min before imaging (Shyu et al., 2008). Additionally, pCXSN-COX4-RFP (Chen et al., 2019) as Mito-Marker constructs and 3NGFP or OsNBL3-GFP fusion constructs were introduced into the Agrobacterium tumefaciens strain EHA105. The constructs were then transformed via A. tumefaciens into Nicotiana benthamiana leaves together using previously described methods (Shyu et al., 2008). Fluorescence was detected $36 \mathrm{~h}$ after infiltration. The primers used are listed in Table S1.

\subsection{RNA isolation and RT-qPCR analysis}

For the expression analysis of defence marker genes and AOX or ND genes, the leaves of five-leaf-stage wild-type and nbl3 plants were used. For the expression pattern analysis, the leaves from the top to bottom, the sheath, stem nodes, panicles and roots of the wild type were used. Total RNA was extracted using the KK Fast Plant Total RNA Kit (Beijing Zoman Biotechnology), and cDNA synthesis was performed using the HiScript II 1st Strand cDNA Synthesis Kit (+gDNA wiper) according to manufacturers' instructions. RT-qPCR was performed using 2x RealStar Green Power Mixture with ROX II and ABI QuantStudio 6 
Flex PCR program, with the rice actin gene (LOC_Os03g50885) used as an internal control. Primers used for the RT-qPCR analyses are listed in Table S1.

\subsection{Circularized RNA reverse transcription PCR (CR-RT-PCR)}

Following the T4 RNA Ligase I (New England Biolabs) guide, $5 \mu \mathrm{g}$ of total RNA from rice leaves at 30 DAS were circularized. First-strand cDNA was synthesized using Prime Script II RTase (TaKaRa) with specific primers (Table S1). The resulting cDNAs were amplified using primers specific to each target gene (Table S1). Each circular RT-PCR fragment was cloned into the pCloneEZ-Blunt TOPO Cloning Kit (Clone Smarter) with ten monoclonals for each target gene sequence.

\subsection{Vector construction and rice transformation}

For the genetic overexpression and RNAi tests, the OsNBL3 coding sequence was amplified using cDNA templates derived from the rice cultivar Aichiasahi. PCR products were cloned into the binary vector pCAMBIA1301 under the control of the maize ubiquitin promoter to generate fused pUbi:3Flag+OsNBL3 constructs. Bases 238 to 797 of the cDNA fragment of OsNBL3 were selected and used for RNAi vector construction. The $386 \mathrm{bp}$ intron of $O s N B L 3$ was used as a linker. The fragments of OsNBL3 were fused into pUbi:OsNBL3-RNAi constructs. The vectors were introduced intoAgrobacterium tumefaciensstrain EHA105 and used to infect wild-type calli. Primers used in the study are listed in Table S1.

\section{RESULTS}

\subsection{Phenotypic characterization of the nbl3 mutant}

The rice mutant nbl3 (natural blight leaf 3 ) was identified in the paddy field containing Geng (japonica) rice cv. Aichiasahi lines that had been subjected to T-DNA insertion. Under field conditions (Beijing), the lower leaves of $n b l 3$ mutants exhibited irregular, brown necrosis lesions at the leaf tip from approximately $30 \mathrm{~d}$ after germination. The lesions then expanded from the tip to the whole leaf, leading to severe leaf withering and premature senescence (Fig. 1a). The cell death lesions gradually occurred from the lower leaves to the upper leaves (Fig. 1b). Up to the heading stage, nbl3 plants exhibited a typical senescence phenotype (Fig. 1c). Under greenhouse conditions, the mutant phenotype of nbl3 occurred later and was less severe than the phenotype that occurred under field conditions. After Trypan blue staining, the nbl3 mutant exhibited dark blue spots, indicating the emergence of cell death or membrane damage on the leaves (Fig. 1d). ROS accumulation has been reported in many LMMs (Chen et al., 2012; Ma et al., 2019; Qiao et al., 2010; S. Wang et al., 2017; Zhao et al., 2020). When DAB and NBT staining were used to conduct the ROS assay, intense brown staining appeared around lesion sites on nbl3 mutant leaves, but no such signal occurred in wild-type leaves (Fig. 1e, f). This indicates that $\mathrm{H}_{2} \mathrm{O}_{2}$ accumulation occurred in thenbl3 leaves. In addition to cell death and premature senescence, several agronomic traits including plant height, tiller number and 1000-grain weight were affected in the nbl3 plants (Fig. S1).

3.2 Enhanced resistance of $n b l 3$ against $M$. oryzaeand Xoo, accompanied by constitutive expression of defence-related genes

The appearance of spontaneous leaf spot is often accompanied by enhanced disease resistance. To determine whether the $n b l 3$ mutation led to enhanced resistance to rice pathogens, one-month-old seedlings of the rice cultivar Aichiasahi (wild type) and the nbl3 mutant were inoculated with $M$. oryzae. The nbl3 plants growing in greenhouse conditions did not display lesions when inoculated with H535, a virulent isolate of $M$. oryzae, using the punch inoculation method. The size of lesions on the nbl3 leaves was significantly smaller than that of lesions on the wild-type leaves at $96 \mathrm{hpi} \mathrm{(Fig.} \mathrm{1g,} \mathrm{h).} \mathrm{Three-month-old} \mathrm{seedlings} \mathrm{were} \mathrm{also}$ inoculated with the bacterial blight pathogen Xoo strain PXO99. It was found that the lesions on the nbl3 leaves were much shorter than those on the wild-type plants 2 weeks post inoculation (Fig. 1i, j). These results demonstrate that nbl3 plants display significantly enhanced resistance to both $M$. oryzae andXoo .

In many rice LMMs, constitutive expression of defence-response genes has been found to be accompanied by lesion development (Ma et al., 2019; Zhao et al., 2020). To determine whether the transcription of defence- 
related genes was affected in thenbl3 mutant, expression analyses were performed using RT-qPCR. Several pathogenesis-related protein genes including OsPR1b , OsPR2, OsPR3, OsPR5, OsPR8 and OsPR10, and two defence-related genes OsWRKY 45 and OsWRKY62, were significantly upregulated in nbl3 (Fig. 2). These results are consistent with the enhanced disease resistance of $n b l 3$. Among these upregulated genes, OsWRKY62 is known to be involved in the jasmonic acid (JA) signalling pathway (Liu et al., 2016), and the other genes are well known to be involved in the salicylic acid (SA) signalling pathway (Tang et al., 2011; Wang et al., 2019). These results suggest that the mutation in nbl3 confers enhanced disease resistance, possibly mediated by both the SA and JA signalling pathways.

\subsection{Cloning of $\mathrm{OsNBL} 3$ and its expression patterns}

Genetic analysis demonstrated that the nbl3 mutation is a recessive trait that is co-segregated with the T-DNA insertion (Table S2). Thereafter, SiteFinding TAIL-PCR procedures were used to isolate chromosomal sequences flanking both ends of the T-DNA. The results showed that the T-DNA was integrated into chromosome 3. According to prediction by The Rice Genome Annotation Project (http://rice.plantbiology.msu.edu/), the insertion site was located in the 3'-untranslated region of an annotated gene LOC_Os03g06370 (Fig. 3a). This insertion event was confirmed by specific PCR using two pairs of primers flanking the insertion site (T-DNA borders; Fig. 3b). Using two pairs of primers to perform the semi-quantitative RT-PCR analysis, the transcript of LOC_Os03g06370 was not detectable in thenbl3 seedlings (Fig. 3c). The RT-qPCR analysis also confirmed that the expression level of LOC_Os03g06370 was dramatically lower in nbl3 than in the wild type (Fig. 3d). These results indicated that the mutation in LOC_Os03g06370 was responsible for the $n b l 3$ phenotypes. This was further confirmed by overexpression

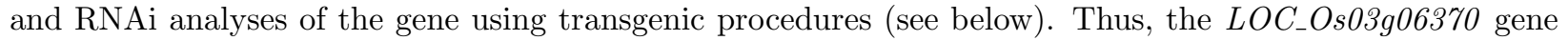
is renamed as OsNBL3 in this study.

Phylogenetic analysis showed that OsNBL3 shared similarity with its homologues in both monocot and dicot plants, and shared especially high identity with homologues in grasses (Fig. S2). To determine the expression patterns of $O s N B L 3$ in rice, RT-qPCR analyses were performed. It was revealed that $O s N B L 3$ was universally expressed in all tested tissues, with relatively high expression in the sheath and roots, and low expression in the basal node and flag leaves at the heading stage (Fig. 3e). In addition, the expression level of OsNBL3 in seedlings was induced by inoculation with the M. oryzae H535 isolate; the induced expression reached a peak at 72 hpi (Fig. 3f).

\subsection{RNAi plants also exhibited enhanced disease resistance}

To further confirm that disruption of $O s N B L 3$ was responsible for the nbl3 phenotypes, we firstly tried to generate knockout lines of OsNBL3 using the CRISPR/Cas9 method (Shan et al., 2013). However, no transgenic plants were obtained through any of several transformation experiments. It is possible that the knockout of $O s N B L 3$ is lethal. Therefore, OsNBL3-RNAi transgenic lines were generated (Fig. 4a), and the transcript levels of $O s N B L 3$ in each homozygous line were confirmed by RT-qPCR analysis (Fig. 4c). Exceeding expectations, the RNAi plants had shorter plant heights similar to those of thenbl3 mutant, while no clear spontaneous cell death was observed on the leaves of the RNAi plants (Fig. S3). To determine whether the RNAi plants exhibited enhanced disease resistance, the resistance of three independent RNAi lines to M. oryzae was tested using the punch inoculation method. The results showed that all the tested lines displayed enhanced resistance (Fig 4b, d). In addition, expression analysis showed that two selected defence-related marker genes, OsPR1b and OsPR5, were significantly upregulated in the RNAi lines (Fig. $\mathrm{S} 4)$. Overexpression (OE) lines of the OsNBL3 gene were also generated (Fig. 4a). In contrast to the RNAi lines, the OE lines did not exhibit obvious reduced or enhanced resistance to $M$. oryzae compared with the wild type (Fig 4b, d). These results further demonstrate that disruption of OsNBL3 is responsible for the enhanced disease resistance.

\subsection{The nbl3 mutant exhibits enhanced salt tolerance}

It has been reported that the mechanism underlying rice LMMs is regulated by hormones and abiotic stresses (Mosher et al., 2010; S. H. Wang et al., 2015; Yamanouchi et al., 2002). To evaluate whether OsNBL3 is 
associated with abiotic stresses, germinated seeds of the $n b l 3$ and wild-type plants were plated on agar plates containing $100 \mathrm{mM} \mathrm{NaCl}$. The root growth of both the $n b l 3$ and wild type were inhibited by $\mathrm{NaCl}$ from 2 to 5 days post treatment. However, the inhibitory rate was significantly lower for $n b l 3$ than for the wild type at each sampling point (Fig. 5a-c). Furthermore, 10-day-old seedlings grown in normal conditions were continuously irrigated with $200 \mathrm{mM} \mathrm{NaCl}$ for twenty days. A greater number of $n b l 3$ plants survived compared to wild-type plants (Fig. 5d, e), which indicates that nbl3 is more tolerant to salt. RT-qPCR analysis was then conducted to determine the expression of $O s N B L 3$ in response to salt treatment. The result showed that $O s N B L 3$ expression was induced by $\mathrm{NaCl}$ with a peak at 10 days post irrigation (Fig. 5f). In addition, the expression analysis showed that the potassium transport gene OsHAK1, the late embryogenesis abundant protein geneOsLEA3, and the transcription factor genes OsNAC22, OsNAP and OsMYB4 , all of which are salt tolerance-related genes (Chen et al., 2015; Chen et al., 2014; Hong, Zhang, Huang, Li, \& Song, 2016; Hu, 2008; Vannini et al., 2006), were significantly upregulated in the nbl3 compared to in the wild type (Fig. $5 \mathrm{~g}$ ). These results demonstrate that $O s N B L 3$ participates in response to salt stress, and disruption of the gene results in enhanced salt tolerance.

\subsection{OsNBL3 is a mitochondria-localized P-type PPR protein}

OsNBL3 encodes a protein with 409 amino acid residues. According to prediction by https://ppr.plantenergy.uwa.edu.au/ (Cheng et al., 2016), OsNBL3 is a PPR protein that harbours seven canonical P-type PPR repeats (Fig. 6a). TargetP prediction analyses showed that the OsNBL3 protein has a mitochondrion-targeting signal at the $\mathrm{N}$ terminus. To determine the subcellular location of OsNBL3, the OsNBL3-GFP construct was made in which the full length cDNA was fused with GFP. The OsNBL3-GFP construct was transformed into the epidermal cells of the $N$. benthamianaleaf, but no GFP signals were observed. It is possible that the entire OsNBL3-GFP protein was difficult to express or was easily degraded. Therefore, another construct (3N-GFP) was made in which the GFP was fused to the N terminus of OsNBL3, harbouring the putative mitochondrion-targeting signal (Fig. 6a). When 3N-GFP was transiently expressed in the epidermal cells of the $N$. benthamiana leaf, the green fluorescent signals of $3 \mathrm{~N}-\mathrm{GFP}$ overlapped with signals from the mitochondria Mito-Marker (Fig. 6b). When $3 \mathrm{~N}-\mathrm{GFP}$ was transiently expressed in rice protoplasts, similar overlays between GFP and signals from the mitochondria dye, Mito-Tracker Red, were also observed (Fig. 6c). These results indicate that OsNBL3 is a mitochondria-localized protein.

\subsection{OsNBL3 participates predominantly in the splicing ofnad5 intron 4}

It had previously been implicated that P-type PPR proteins participate in plant organelle RNA metabolism, including 5' processing, intron splicing and translation (Barkan \& Small, 2014). Given that OsNBL3 is a mitochondrion-targeting protein, CR-RT-PCR procedures were first used to examine whether the 5' processing of respiratory complex genes was affected in the nbl3mutant. Among 17 analysed genes, the amplified products of each gene in the wild type and nbl3 were the same size (Fig S5). This suggested that 5' processing was not affected by the mutation in $n b l 3$. Next, the mature transcripts of 34 mitochondrial genes were examined using total RNA from 30-day-old seedlings of the wild-type and $n b l 3$ plants with specific primers (Fig. 7a). The results showed that only the mature nad5 transcript was obviously reduced in $n b l 3$.

There are four introns in the nad5 gene, including twocis -introns and two trans -introns (Bonen, 2008). To determine whether the reduction of the nad5 transcript resulted from splicing defects in nbl3 , RT-qPCR was conducted to analyse the ratio of spliced to unspliced transcripts of all the 23 introns in mitochondria. The splicing efficiency of nad5 intron 4 was dramatically reduced in nbl3 compared to in the wild type (Fig. $7 \mathrm{~b})$. To further verify improper splicing of $n a d 5$ in $n b l 3$, reverse transcription PCR was performed, allowing amplification across adjacent exons and detection of each splicing event in nad5 (Fig. 7c). The amount of cis -spliced transcripts of exons $4-5$ (exon $4+$ exon 5) was greatly reduced in the nbl3 mutant (Fig. 7d). These results suggest that OsNBL3 is required for the cis -splicing of the mitochondrial nad5 intron 4. Interestingly, the amount oftrans -spliced transcripts of exons $2-4$ (exon $2+$ exon $3+$ exon 4 ) was also weakly reduced in the nbl3 mutant, suggesting that OsNBL3 also participates in the trans -splicing of nad5 in mitochondria. 


\subsection{OsNBL3 mutation affects mitochondria morphology andelevates alternative respiratory pathways}

NAD5 is a subunit of NADH dehydrogenase (complex I) in the mitochondrial respiratory chain. To investigate whether mitochondrial morphology was affected by the disruption of $O s N B L 3$, transmission electron microscopy assays were used to observe the ultrastructure of the mitochondria in mesophyll cells from eightweek-old $n b l 3$ and wild-type plant leaves. The overall number of mitochondria was lower in $n b l 3$ than in the wild type, and the overall size of mitochondria was relatively greater in nbl3 . Specifically, swelling cristae with vesicle-like structures and reduced intermembrane content were observed in the $n b l 3$ mesophyll cells (Fig. 8a and Fig S6).

Disruption of the ETC in plant mitochondria usually leads to the induction of alternative respiratory pathway genes that are known to be mitochondrial stress markers (Vanlerberghe, 2013). RT-qPCR analysis showed that there were significantly more $A O X 1 a$ and $A O X 1 \mathrm{ctranscripts}$ in the leaves of 30-day-old nbl3 seedlings than in the leaves of wild-type seedlings (Fig. 8b). Plant mitochondria have internal and external NADH dehydrogenases that function as alternative dehydrogenases (Rasmusson \& Wallstrom, 2010). Expression analysis showed that the internal NADH dehydrogenase genes, NDB2 and NDB3, and the external NADH dehydrogenase genes, $N D A 1$ and $N D C 1$, were expressed at a higher level in the leaves of four-week-old nbl3 seedlings than in four-week-old wild-type seedlings (Fig. 8b). These results demonstrate that loss of OsNBL3 function affects the assembly of complex I, leading to the induction of the alternative respiratory pathway.

\section{DISCUSSION}

\subsection{The nbl3 mutant is a new $s p l$ mutant caused by the disruption of a PPR gene}

Plant cell death and structural adaptations may protect plants from biotic and abiotic stresses, including pathogens, salt and high temperature, but excluding ROS (Ma et al., 2019; Yamanouchi et al., 2002; Zeng et al., 2004). The molecular mechanism underlying plant cell death and defence responses in LMMs orspl mutants have been partially elucidated. spl mutants show spontaneous HR-like necrotic lesions and bursts of ROS, and activate the expression of defence genes (S. Wang et al., 2014; Xu et al., 2014). In this study,nbl3 exhibited growth retardation, leaf wilting and premature senescence (Fig. 1a-c). Interestingly, unlike most identified splmutants, that usually display regular-shaped reddish-brown spots, the lesion mimic spots of $n b l 3$ only occurred at the seedling stage. From the tilling stage, the leaves of nbl3 gradually withered from the lower to upper leaves, without obvious dark spots. Histochemical staining using Trypan blue, NBT and DAB showed that thenbl3 mutant displayed ROS accumulation and cell death in the leaves (Fig. 1d-f). These symptoms have also been observed in otherspl mutants (S. Wang et al., 2017; Zhao et al., 2020). These results indicated that $n b l 3$ is a new $s p l$ mutant.

In addition to ROS bursts and cell death, many spl mutants (LMMs) exhibit enhanced disease resistance (Chen et al., 2012; Ma et al., 2019; Qiao et al., 2010; Yamanouchi et al., 2002). In the present study, it was found that the nbl3 mutant showed enhanced resistance against both fungal and bacterial pathogens (Fig. 1g-j). This suggests that OsNBL3 may act as a negative regulator in PCD and resistance signalling pathways in plants (Fig. 2, 3f). Interestingly, the RNAi plants did not show spontaneous cell death in the same way that the nbl3 mutant did. However, the RNAi plants did display enhanced resistance to $M$. oryzae (Fig. 4b, d). An explanation for this could be that the transcripts were destroyed in the nbl3mutant by a T-DNA insertion within the 3'-untranslated region of OsNBL3. Meanwhile, the downregulation of OsNBL3 in the RNAi plants was mediated via a post-transcriptional silencing mechanism, which allowed for a few intact transcripts to be present. In addition, the nbl3 mutant showed enhanced salt tolerance (Fig. 5). To our knowledge, nbl3 is a newly characterized $s p l$ mutant that possesses enhanced salt tolerance.

OsNBL3 encodes a mitochondria-localized PPR protein (Fig. 6b, c). As RNA binding factors, PPR proteins regulate the RNA expression in organelles, thereby affecting plant growth and development. There are many reports involving PPR mutants that have shown that PPR proteins are involved in regulating the physiological functions of plant growth and development. Such PPR mutants have displayed cytoplasmic male sterility (CMS) (Hu et al., 2012; Huang et al., 2015), defective seed or embryo development (Liu et al., 
2020; G. Wang et al., 2017), restricted plant growth (Xie et al., 2016; Zhu et al., 2012), organelle development defects (Lin et al., 2015; Z. W. Wang et al., 2018), albino leaf phenotype (Su et al., 2012; Tang et al., 2017), and insensitivity to abiotic stress (Yuan \& Liu, 2012; Zsigmond et al., 2012). In this study,nbl3 showed almost all of the physiological phenotypes of PPR mutants, such as growth retardation (Fig. 1a), reduced seed setting rate (Fig. S1) and enhanced salt tolerance (Fig. 5). However, unlike the other PPR mutants, the gene responsible for the $n b l 3$ phenotype is the first mitochondria-localized PPR gene to be described and is involved in the regulation of rice disease resistance pathways.

\subsection{OsNBL3 is involved in splicing nad5 introns}

PPR proteins are usually encoded by nuclear genes and then transported to organelles to regulate RNA metabolism, including intron splicing, RNA editing, 5'- and 3' - modification, RNA degradation and translation and other post-transcriptional modification processes (Barkan \& Small, 2014). It is well known that mitochondria provide energy for cell processes. Group II introns are ubiquitous in the organelle genomes of flowering plants. There are 23 group II introns in rice mitochondria, 19 of which are distributed in the genes encoding subunits of the NADH dehydrogenase complex (complex I) (Bonen, 2008). Complex I is the first enzyme complex in the mitochondrial respiratory chain, the starting point for electrons to enter the respiratory chain, and essential for mitochondrial energy production and electron transfer (Dai et al., 2018; Wu et al., 2019). It has been reported that defects in the intron splicing of nad mRNAs could lead to partial or complete reductions in the activity of ETC complex I, and disturb plant growth and development. Recently, several P-type PPR proteins have been identified as splicing factors of nad in Arabidopsis and maize. Defects in nad gene intron splicing result in a significant decrease in the assembly and activity of complex I and overexpression of AOX genes in the misf and ppr19 mutants of Arabidopsis thaliana (Lee et al., 2017; Wang, Aube, Quadrado, Dargel-Graffin, \& Mireau, 2018). The maize mutants dek37, emp12 and ppr20 displayed compromised splicing efficiencies of nad2 introns, and mitochondrial morphology and seed development was also affected (Dai et al., 2018; Sun et al., 2019; Yang et al., 2020). Furthermore, DEK41 and EMP602 have been shown to be required for the splicing ofnad/4 introns and seed development (Ren et al., 2019; Zhu et al., 2019). ZmSMK9 affected the development of the kernel and plant architecture by participating in the splicing of nad5 introns in maize (Pan et al., 2019). In rice, FLO10and $R L 1$ were reported to be involved in the splicing ofnad1 intron 1 and nad4 intron 1, respectively (Wu et al., 2019; Wu et al., 2020). The nad5contains four group II introns, of which introns 1 and 4 arecis -spliced, while introns 2 and 3 are trans -spliced (Yang, et al. 2020). In the present study, it was found that the disruption of OsNBL3 compromised the splicing of nad5introns in mitochondria (Fig. 7b-d). The mutation of OsNBL3 also affected the mitochondrial morphology with the collapse of cristae with vesicle-like structures, and elevated reliance on alternative respiratory pathways (Fig. 8a, b and Fig. S5). The destruction of the inner mitochondrial membrane underlies the production of mtROS and results in their leakage into the cytoplasm. Moreover, the leakage of mtROS further destroys the intracellular environment and homeostasis, which via feedback, activates the plant's protective mechanism and triggers local ROS elevation and HR response, as well as growth retardation and pre-senescence. Overall, it is plausible that the mutation of the OsNBL3 gene is responsible for the $n b l 3$ phenotype as the fully functioning gene normally participates in the splicing of nad5 introns. The results provide OsNBL3 as a new regulator for cell death, thus contributing to explaining the mechanisms underlying plant defence against biotic and abiotic stresses.

\section{Acknowledgements}

This work was supported by grants from the National Natural Science Foundation of China (Grant No. 31872926) and the National Key Research and Development Program of China (Grant No. 2016YFD0100601). We thank Professor Yuese Ning from the Chinese Academy of Agricultural Sciences for his kind gift with the plasmid pCXSN-COX4-RFP.

\section{References}

Barkan, A., \& Small, I. (2014). Pentatricopeptide repeat proteins in plants. Annu Rev Plant Biol, 65 (1), 415. 
Blokhina, O., \& Fagerstedt, K. V. (2010). Reactive oxygen species and nitric oxide in plant mitochondria: origin and redundant regulatory systems. Physiol Plant, 138 (4), 447-462.

Bonen, L. (2008). Cis- and trans-splicing of group II introns in plant mitochondria. Mitochondrion, 8 (1), 26-34.

Chen, G., Hu, Q., Luo, L., Yang, T., Zhang, S., Hu, Y., . . . Xu, G. (2015). Rice potassium transporter OsHAK1 is essential for maintaining potassium-mediated growth and functions in salt tolerance over low and high potassium concentration ranges. Plant Cell Environ, 38 (12), 2747-2765.

Chen, G. L., Zou, Y., Hu, J. H., \& Ding, Y. (2018). Genome-wide analysis of the rice PPR gene family and their expression profiles under different stress treatments. Bmc Genomics, 19 .

Chen, X., Wang, Y. F., Lv, B., Li, J., Luo, L. Q., Lu, S. C., . . . Ming, F. (2014). The NAC Family Transcription Factor OsNAP Confers Abiotic Stress Response Through the ABA Pathway. Plant And Cell Physiology, 55 (3), 604-619.

Chen, X. F., Hao, L., Pan, J. W., Zheng, X. X., Jiang, G. H., Jin, Y., . . . Ma, B. J. (2012). SPL5, a cell death and defense-related gene, encodes a putative splicing factor $3 \mathrm{~b}$ subunit 3 (SF3b3) in rice.Molecular Breeding, 30 (2), 939-949.

Chen, Z., Zheng, W., Chen, L., Li, C., Liang, T., Chen, Z., .. \& \& Wang, F. (2019). Green fluorescent proteinand Discosoma sp. red fluorescent protein-tagged organelle marker lines for protein subcellular localization in rice. Frontiers in plant science, 10.

Cheng, S., Gutmann, B., Zhong, X., Ye, Y., Fisher, M. F., Bai, F., . . . Huang, J. (2016). Redefining the structural motifs that determine RNA binding and RNA editing by pentatricopeptide repeat proteins in land plants. Plant Journal, 85 (4), 532.

Colas des Francs-Small, C., Falcon de Longevialle, A., Li, Y., Lowe, E., Tanz, S. K., Smith, C., . . . Small, I. (2014). The Pentatricopeptide Repeat Proteins TANG2 and ORGANELLE TRANSCRIPT PROCESSING439 Are Involved in the Splicing of the Multipartite nad5 Transcript Encoding a Subunit of Mitochondrial Complex I. Plant Physiol, 165 (4), 1409-1416.

Colombatti, F., Gonzalez, D. H., \& Welchen, E. (2014). Plant mitochondria under pathogen attack: a sigh of relief or a last breath? Mitochondrion, 19 Pt B , 238-244.

Dai, D. W., Luan, S. C., Chen, X. Z., Wang, Q., Feng, Y., Zhu, C. G., . . . Song, R. T. (2018). Maize Dek37 Encodes a P-type PPR Protein That Affects cis-Splicing of Mitochondrial nad2 Intron 1 and Seed Development. Genetics, 208 (3), 1069-1082.

De Longevialle, A. F., Meyer, E. H., Andres, C., Taylor, N. L., Lurin, C., Millar, A. H., \& Small, I. D. (2007). The pentatricopeptide repeat gene OTP43 is required for trans-splicing of the mitochondrial nad1 intron 1 in Arabidopsis thaliana. Plant Cell, 19 (10), 3256-3265.

Diaz, M. F., Bentolila, S., Hayes, M. L., Hanson, M. R., \& Mulligan, R. M. (2017). A protein with an unusually short PPR domain, MEF8, affects editing at over 60 Arabidopsis mitochondrial C targets of RNA editing.Plant Journal, 92 (4), 638-649.

Fang, W. W., Liu, C. C., Zhang, H. W., Xu, H., Zhou, S., Fang, K. X., . . . Zhao, W. S. (2018). Selection of Differential Isolates of Magnaporthe oryzae for Postulation of Blast Resistance Genes.Phytopathology, 108 (7), 878-884.

Fekih, R., Tamiru, M., Kanzaki, H., Abe, A., Yoshida, K., Kanzaki, E., . . . Terauchi, R. (2015). The rice (Oryza sativa L.) LESION MIMIC RESEMBLING, which encodes an AAA-type ATPase, is implicated in defense response. Molecular Genetics and Genomics, 290 (2), 611-622.

Greenberg, J. T., Guo, A., Klessig, D. F., \& Ausubel, F. M. (1994). Programmed cell death in plants: a pathogen-triggered response activated coordinately with multiple defense functions. Cell, 77 (4), 551-563. 
Haili, N., Arnal, N., Quadrado, M., Amiar, S., Tcherkez, G., Dahan, J., . . . Mireau, H. (2013). The pentatricopeptide repeat MTSF1 protein stabilizes the nad4 mRNA in Arabidopsis mitochondria. Nucleic Acids Res, 41 (13), 6650-6663.

Haili, N., Planchard, N., Arnal, N., Quadrado, M., Vrielynck, N., Dahan, J., . . . Mireau, H. (2016). The MTL1 Pentatricopeptide Repeat Protein Is Required for Both Translation and Splicing of the Mitochondrial NADH DEHYDROGENASE SUBUNIT7 mRNA in Arabidopsis. Plant Physiol, 170 (1), 354-366.

Hong, Y. B., Zhang, H. J., Huang, L., Li, D. Y., \& Song, F. M. (2016). Overexpression of a Stress-Responsive NAC Transcription Factor Gene ONACO22 Improves Drought and Salt Tolerance in Rice. Front Plant Sci, 7 .

Hsieh, W. Y., Liao, J. C., Chang, C. Y., Harrison, T., Boucher, C., \& Hsieh, M. H. (2015). The SLOW GROWTH3 Pentatricopeptide Repeat Protein Is Required for the Splicing of Mitochondrial NADH Dehydrogenase Subunit7 Intron 2 in Arabidopsis. Plant Physiol, 168 (2), 490-+.

Hu, J., Wang, K., Huang, W., Liu, G., Gao, Y., Wang, J., . . . Zhu, Y. (2012). The rice pentatricopeptide repeat protein RF5 restores fertility in Hong-Lian cytoplasmic male-sterile lines via a complex with the glycine-rich protein GRP162. Plant Cell, 24 (1), 109-122.

$\mathrm{Hu}$, T. Z. (2008). OsLEA3, a late embryogenesis abundant protein gene from rice, confers tolerance to water deficit and salt stress to transgenic rice. Russian Journal of Plant Physiology, 55 (4), 530-537.

Huang, S., Van Aken, O., Schwarzlander, M., Belt, K., \& Millar, A. H. (2016). The Roles of Mitochondrial Reactive Oxygen Species in Cellular Signaling and Stress Response in Plants. Plant Physiol, 171 (3), 15511559 .

Huang, W. C., Yu, C. C., Hu, J., Wang, L. L., Dan, Z. W., Zhou, W., . . . Zhu, Y. G. (2015). Pentatricopeptide-repeat family protein RF6 functions with hexokinase 6 to rescue rice cytoplasmic male sterility.Proc Natl Acad Sci U S A, 112 (48), 14984-14989.

Ishikawa, A., Okamoto, H., Iwasaki, Y., \& Asahi, T. (2001). A deficiency of coproporphyrinogen III oxidase causes lesion formation in Arabidopsis. Plant J, 27 (2), 89-99.

Jones, A. M., \& Dangl, J. L. (1996). Logjam at the Styx: programmed cell death in plants. Trends Plant Sci, 1 (4), 114-119.

Lee, K., Han, J. H., Park, Y. I., des Francs-Small, C. C., Small, I., \& Kang, H. (2017). The mitochondrial pentatricopeptide repeat protein PPR19 is involved in the stabilization of NADH dehydrogenase 1 transcripts and is crucial for mitochondrial function and Arabidopsis thaliana development. New Phytologist, 215 (1), 202-216.

Li, J., Brader, G., \& Palva, E. T. (2004). The WRKY70 transcription factor: a node of convergence for jasmonate-mediated and salicylate-mediated signals in plant defense. The Plant Cell, 16(2), 319-331.

Li, X. J., Gu, W., Sun, S. L., Chen, Z. L., Chen, J., Song, W. B., . . . Lai, J. S. (2018). Defective Kernel 39 encodes a PPR protein required for seed development in maize. J Integr Plant Biol, 60 (1), 45-64.

Liang, H., Yao, N., Song, L. T., Luo, S., Lu, H., \& Greenberg, L. T. (2003). Ceramides modulate programmed cell death in plants. Genes \& Development, 17 (21), 2636-2641.

Lin, D., Gong, X., Quan, J., Zheng, K., Hua, Z., Xu, J., . . . Dong, Y. (2015). The rice ALS3 encoding a novel pentatricopeptide repeat protein is required for chloroplast development and seedling growth. Rice, 8 (1), 17.

Liu, J., Chen, X. , Liang, X., Zhou, X. , Yang, F. , \& Liu, J. , et al. (2016). Alternative splicing of rice wrky62 and wrky76 transcription factor genes in pathogen defense. Plant Physiology, pp.01921.2015. 
Liu, R., Cao, S. K., Sayyed, A., Yang, H. H., Zhao, J., Wang, X., . . . Tan, B. C. (2020). The DYW-subgroup pentatricopeptide repeat protein PPR27 interacts with ZmMORF1 to facilitate mitochondrial RNA editing and seed development in maize. J Exp Bot, 71 (18), 5495-5505.

Lorrain, S., Lina, B. Q., Auriac, M. C., Kroj, T., Saindrenan, P., Nicole, M., . . . Roby, D. (2004). Vascular associated death1, a novel gram domain-containing protein, is a regulator of cell death and defense responses in vascular tissues. Plant Cell, 16 (8), 2217-2232.

Lyu, L., Bi, Y., Li, S. G., Xue, H. L., Zhang, Z., \& Prusky, D. B. (2019). Early Defense Responses Involved in Mitochondrial Energy Metabolism and Reactive Oxygen Species Accumulation in Harvested Muskmelons Infected by Trichothecium roseum. Journal Of Agricultural And Food Chemistry, 67 (15), 4337-4345.

Ma, J., Wang, Y., Ma, X., Meng, L., Jing, R., Wang, F., . . . Wan, J. (2019). Disruption of gene SPL35, encoding a novel CUE domain-containing protein, leads to cell death and enhanced disease response in rice.Plant Biotechnol J, 17 (8), 1679-1693.

Mosher, S., Moeder, W., Nishimura, N., Jikumaru, Y., Joo, S. H., Urquhart, W., . . . Yoshioka, K. (2010). The Lesion-Mimic Mutant cpr22 Shows Alterations in Abscisic Acid Signaling and Abscisic Acid Insensitivity in a Salicylic Acid-Dependent Manner. Plant Physiol, 152 (4), 1901-1913.

Noutoshi, Y., Kuromori, T., Wada, T., Hirayama, T., Kamiya, A., Imura, Y., . . . Shinozaki, K. (2006). Loss of Necrotic Spotted Lesions 1 associates with cell death and defense responses in Arabidopsis thaliana. Plant Mol Biol, 62 (1-2), 29-42.

Pan, Z. Y., Liu, M., Xiao, Z. Y., Ren, X. M., Zhao, H. L., Gong, D. M., . . . Qiu, F. (2019). ZmSMK9, a pentatricopeptide repeat protein, is involved in the cis-splicing of nad5, kernel development and plant architecture in maize. Plant Science, 288 .

Poor, P., Patyi, G., Takacs, Z., Szekeres, A., Bodi, N., Bagyanszki, M., \& Tari, I. (2019). Salicylic acidinduced ROS production by mitochondrial electron transport chain depends on the activity of mitochondrial hexokinases in tomato (Solanum lycopersicum L.). J Plant Res, 132 (2), 273-283.

Qiao, Y. L., Jiang, W. Z., Joohyun, L., Bongsoo, P., Minseon, C., Rihua, P., . . . Namchon, P. (2010). SPL28 encodes a clathrin-associated adaptor protein complex 1, medium subunit $\mu 1$ (AP1M1) and is responsible for spotted leaf and early senescence in rice (Oryza sativa). New Phytologist, 185 (1), 258-274.

Rasmusson, A. G., \& Wallstrom, S. V. (2010). Involvement of mitochondria in the control of plant cell $\mathrm{NAD}(\mathrm{P}) \mathrm{H}$ reduction levels. Biochemical Society Transactions, 38 , 661-666.

Ren, Z., Fan, K., Fang, T., Zhang, J., Yang, L., Wang, J., . . . Liu, Y. (2019). Maize Empty Pericarp602 Encodes a P-Type PPR Protein That Is Essential for Seed Development. Plant And Cell Physiology, 60 (8), 1734-1746.

Rostoks, N., Schmierer, D., Mudie, S., Drader, T., Brueggeman, R., Caldwell, D. G., . . . Kleinhofs, A. (2006). Barley necrotic locus nec1 encodes the cyclic nucleotide-gated ion channel 4 homologous to the Arabidopsis HLM1. Mol Genet Genomics, 275 (2), 159-168.

Schmitzlinneweber, C., \& Small, I. (2008). Pentatricopeptide repeat proteins: a socket set for organelle gene expression. Trends Plant Sci, 13 (12), 663-670.

Shan, Q., Wang, Y., Li, J., Zhang, Y., Chen, K., Liang, Z., .. \& Gao, C. (2013). Targeted genome modification of crop plants using a CRISPR-Cas system. Nature biotechnology, 31 (8), 686-688.

Shyu, Y. J., Hiatt, S. M., Duren, H. M., Ellis, R. E., Kerppola, T. K., \& Hu, C. D. (2008). Visualization of protein interactions in living Caenorhabditis elegans using bimolecular fluorescence complementation analysis. Nature Protocols, 3 (4), 588-596.

Su, N., Hu, M. L., Wu, D. X., Wu, F. Q., Fei, G. L., Lan, Y., . . . Wan, J. M. (2012). Disruption of a rice pentatricopeptide repeat protein causes a seedling-specific albino phenotype and its utilization to enhance 
seed purity in hybrid rice production. Plant Physiol, 159 (1), 227-238.

Sun, F., Xiu, Z. H., Jiang, R. C., Liu, Y. W., Zhang, X. Y., Yang, Y. Z., . . . Tan, B. C. (2019). The mitochondrial pentatricopeptide repeat protein EMP12 is involved in the splicing of three nad2 introns and seed development in maize. J Exp Bot, 70 (3), 963-972.

Sungryul, K., Yang, J. I., Sunok, M., Choonghwan, R., An, K. S., Kyungme, K., . . . An, G. H. (2009). Rice OGR1 encodes a pentatricopeptide repeat-DYW protein and is essential for RNA editing in mitochondria. Plant Journal for Cell \& Molecular Biology, 59 (5), 738-749.

Suzuki, N., Koussevitzky, S., Mittler, R., \& Miller, G. (2012). ROS and redox signalling in the response of plants to abiotic stress.Plant Cell Environ, 35 (2), 259-270.

Tan, G. H., Gao, Y., Shi, M., Zhang, X. Y., He, S. P., Cheng, Z. L., \& An, C. C. (2005). SiteFinding-PCR: a simple and efficient PCR method for chromosome walking. Nucleic Acids Res, 33 (13).

Tanaka, R., Hirashima, M., Satoh, S., \& Tanaka, A. (2003). The Arabidopsis-accelerated cell death gene ACD1 is involved in oxygenation of pheophorbide a: Inhibition of the pheophorbide a oxygenase activity does not lead to the "Stay-Green" phenotype in Arabidopsis. Plant And Cell Physiology, 44 (12), $1266-1274$.

Tang, J. P., Zhang, W. W., Wen, K., Chen, G. M., Sun, J., Tian, Y. L., . . . Wan, J. M. (2017). OsPPR6, a pentatricopeptide repeat protein involved in editing and splicing chloroplast RNA, is required for chloroplast biogenesis in rice. Plant Mol Biol, 95 (4-5), 345-357.

Tang, J. Y., Zhu, X. D., Wang, Y. Q., Liu, L. C., Xu, B., Li, F., . . . Chu, C. C. (2011). Semi-dominant mutations in the CC-NB-LRR-type R gene, NLS1, lead to constitutive activation of defense responses in rice.Plant Journal, 66 (6), 996-1007.

Toda, T., Fujii, S. , Noguchi, K., Kazama, T., \& Toriyama, K. (2012). Rice MPR25 encodes a pentatricopeptide repeat protein and is essential for RNA editing of nad5 transcripts in mitochondria. Plant Journal, 72 (3), 450-460.

Undan, J. R., Tamiru, M., Abe, A., Yoshida, K., Kosugi, S., Takagi, H., . . . Terauchi, R. (2012). Mutation in OsLMS, a gene encoding a protein with two double-stranded RNA binding motifs, causes lesion mimic phenotype and early senescence in rice (Oryza sativa L.). Genes Genet Syst, 87 (3), 169-179.

Van Aken, O., \& Van Breusegem, F. (2015). Licensed to Kill: Mitochondria, Chloroplasts, and Cell Death. Trends Plant Sci, 20 (11), 754-766.

Vanlerberghe, G. C. (2013). Alternative oxidase: a mitochondrial respiratory pathway to maintain metabolic and signaling homeostasis during abiotic and biotic stress in plants. Int J Mol Sci, 14 (4), 6805-6847.

Vannini, C., Iriti, M., Bracale, M., Locatelli, F., Faoro, F., Croce, P., . . . Genga, A. (2006). The ectopic expression of the rice Osmyb4 gene in Arabidopsis increases tolerance to abiotic, environmental and biotic stresses. Physiological And Molecular Plant Pathology, 69 (1-3), 26-42.

Wang, C. D., Aube, F., Planchard, N., Quadrado, M., Dargel-Graffin, E., Nogue, F., \& Mireau, H. (2017). The pentatricopeptide repeat protein MTSF2 stabilizes a nad1 precursor transcript and defines the 3 ' end of its 5 '-half intron. Nucleic Acids Res, 45 (10), 6119-6134.

Wang, C. D., Aube, F., Quadrado, M., Dargel-Graffin, C., \& Mireau, H. (2018). Three new pentatricopeptide repeat proteins facilitate the splicing of mitochondrial transcripts and complex I biogenesis in Arabidopsis. J Exp Bot, 69 (21), 5131-5140.

Wang, G., Zhong, M. Y., Shuai, B. L., Song, J. D., Zhang, J., Han, L., . . . Song, R. T. (2017). E plus subgroup PPR protein defective kernel 36 is required for multiple mitochondrial transcripts editing and seed development in maize and Arabidopsis. New Phytologist, 214 (4), 1563-1578. 
Wang, L., Pei, Z., Tian, Y., \& He, C. (2005). OsLSD1, a rice zinc finger protein, regulates programmed cell death and callus differentiation. Mol Plant Microbe Interact, 18 (5), 375-384.

Wang, M. , Yang, D. , Ma, F. , Zhu, M. , Shi, Z. , \& Miao, X. . (2019). Oshlh61-osbhlh96 influences rice defense to brown planthopper through regulating the pathogen-related genes. Rice (New York, N.Y.), 12(1), 9 .

Wang, S., Lei, C., Wang, J., Ma, J., Tang, S., Wang, C., . . . Qi, C. (2017). SPL33, encoding an eEF1A-like protein, negatively regulates cell death and defense responses in rice. J Exp Bot, 68 (5), 899-913.

Wang, S. H., Lim, J. H., Kim, S. S., Cho, S. H., Yoo, S. C., Koh, H. J., . . . Paek, N. C. (2015). Mutation of SPOTTED LEAF3 (SPL3) impairs abscisic acid-responsive signalling and delays leaf senescence in rice. $J$ Exp Bot, 66 (22), 7045.

Wang, Z., Wang, Y., Hong, X., Hu, D., Liu, C., Yang, J., . . . Li, Y. (2015). Functional inactivation of UDP-N-acetylglucosamine pyrophosphorylase 1 (UAP1) induces early leaf senescence and defence responses in rice. J Exp Bot, 66 (3), 973-987.

Wang, Z. W., Lv, J., Xie, S. Z., Zhang, Y., Qiu, Z. N., Chen, P., . . . Ren, D. Y. (2018). OsSLA4 encodes a pentatricopeptide repeat protein essential for early chloroplast development and seedling growth in rice.Plant Growth Regulation, 84 (2), 249-260.

Weissenberger, S., Soll, J., \& Carrie, C. (2017). The PPR protein SLOW GROWTH 4 is involved in editing of nad4 and affects the splicing of nad2 intron 1. Plant Mol Biol, 93 (4-5), 355-368.

Wu, J., Sun, Y., Zhao, Y., Zhang, J., Luo, L., Li, M., . . . Li, J. (2015). Deficient plastidic fatty acid synthesis triggers cell death by modulating mitochondrial reactive oxygen species. Cell Res, 25 (5), 621-633.

Wu, M. M., Ren, Y. L., Cai, M. H., Wang, Y. L., Zhu, S. S., Zhu, J. P., . . . Wan, J. M. (2019). Rice FLOURY ENDOSPERM10 encodes a pentatricopeptide repeat protein that is essential for the trans-splicing of mitochondrial nad1 intron 1 and endosperm development.New Phytologist, 223 (2), 736-750.

Wu, M. W., Zhao, H., Zhang, J. D., Guo, L., \& Liu, C. M. (2020). RADICLELESS 1 (RL1)-mediated nad4 intron 1 splicing is crucial for embryo and endosperm development in rice (Oryza sativa L.).Biochemical And Biophysical Research Communications, 523 (1), 220-225.

Xie, T., Chen, D., Wu, J., Huang, X., Wang, Y., Tang, K., . . . Peng, X. (2016). Growing Slowly 1 locus encodes a PLS-type PPR protein required for RNA editing and plant development in Arabidopsis. J Exp Bot, 67 (19), 5687-5698.

Xu, X., Zhang, L. L., Liu, B. M., Ye, Y. F., \& Wu, Y. J. (2014). Characterization and mapping of a spotted leaf mutant in rice (Oryza sativa). Genetics And Molecular Biology, 37 (2), 406-413.

Yamanouchi, U., Yano, M., Lin, H., Ashikari, M., \& Yamada, K. (2002). A rice spotted leaf gene, Spl7, encodes a heat stress transcription factor protein. Proc Natl Acad Sci U S A, 99 (11), 7530.

Yang, M. L., Wardzala, E., Johal, G. S., \& Gray, J. (2004). The wound-inducible Lls1 gene from maize is an orthologue of the Arabidopsis Acd1 gene, and the LLS1 protein is present in non-photosynthetic tissues. Plant Mol Biol, 54 (2), 175-191.

Yang, Y. Z., Ding, S., Wang, Y., Wang, H. C., Liu, X. Y., Sun, F., . . . Tan, B. C. (2020). PPR20 Is Required for the cis-Splicing of Mitochondrial nad2 Intron 3 and Seed Development in Maize. Plant And Cell Physiology, 61 (2), 370-380.

Yuan, H., \& Liu, D. (2012). Functional disruption of the pentatricopeptide protein SLG1 affects mitochondrial RNA editing, plant development, and responses to abiotic stresses in Arabidopsis.Plant Journal, 70 (3), 432-444. 
Zeng, L. R., Qu, S., Bordeos, A., Yang, C., Baraoidan, M., Yan, H., . . . Wang, G. L. (2004). Spotted leaf11, a negative regulator of plant cell death and defense, encodes a U-box/armadillo repeat protein endowed with E3 ubiquitin ligase activity. Plant Cell, 16 (10), 2795.

Zhang, Y. F., Suzuki, M., Sun, F., \& Tan, B. C. (2017). The Mitochondrion-Targeted PENTATRICOPEPTIDE REPEAT78 Protein Is Required for nad5 Mature mRNA Stability and Seed Development in Maize. Mol Plant, 10 (10), 1321-1333.

Zhao, X., Qiu, T., Feng, H., Yin, C., Zheng, X., Yang, J., . . . Zhao, W. (2020). A Novel Glycine-Rich Domain Protein, OsGRDP1, Functions as a Critical Feedback Regulator for Controlling Cell Death and Disease Resistance in Rice. J Exp Bot . doi: 10.1093/jxb/eraa450

Zhu, C. G., Jin, G. P., Fang, P., Zhang, Y., Feng, X. Z., Tang, Y. P., . . . Song, R. T. (2019). Maize pentatricopeptide repeat protein DEK41 affects cis-splicing of mitochondrial nad4 intron 3 and is required for normal seed development. J Exp Bot, 70 (15), 3795-3808.

Zhu, Q., Dugardeyn, J., Zhang, C. Y., Takenaka, M., Kuhn, K., Craddock, C., . . . Van Der Straeten, D. (2012). SLO2, a mitochondrial pentatricopeptide repeat protein affecting several RNA editing sites, is required for energy metabolism. Plant Journal, 71 (5), 836-849.

Zsigmond, L., Szepesi, A., Tari, I., Rigo, G., Kiraly, A., \& Szabados, L. (2012). Overexpression of the mitochondrial PPR40 gene improves salt tolerance in Arabidopsis. Plant Science, 182, 87-93.

\section{Figure legends}

\section{FIGURE 1}

The nbl3 mutant exhibits a lesion mimic phenotype accompanied by a reactive oxygen species burst, accelerated leaf senescence and enhanced disease resistance. (a) Whole wild-type (WT) and $n b l 3$ mutant plants at the flowering stage in the paddy field. Scale bars $=10.0 \mathrm{~cm}$. (b) Lesion mimic phenotypes on different leaves of the nbl3 mutant at the pollination stage, compared to that of the wild type. The lower to upper leaves were arranged from right to left. (c) The second leaf of the wild type and the $n b l 3$ mutant. (d-f) Photographs of leaves stained with Trypan blue, tetranitroblue tetrazolium chloride (NBT) solution and 3,3'-diaminobenzidine (DAB). (g) Lesion lengths of the inoculated leaves at $96 \mathrm{~h}$ post punch inoculation with isolate H535 of Magnaporthe oryzae. Data are shown as means $\pm \mathrm{SD}, \mathrm{n}=10$ $\left({ }^{* *} P<0.01\right.$; Student's $t$ test). (h) Leaves of the wild type and $n b l 3$ mutant were photographed at $96 \mathrm{~h}$ post inoculation with $M$. oryzae isolate H535. (i) Lesion lengths of the inoculated leaves at two weeks' post inoculation withXanthomonas oryzae pv. oryzae (Xoo ) strain PXO99. Data were shown as means $\pm \mathrm{SD}, \mathrm{n}$ $=15\left({ }^{* *} P<0.01\right.$; Student's $t$ test). (j) Leaves of the wild type and nbl3mutant were photographed at two weeks post inoculation with Xoostrain PXO99.

\section{FIGURE 2}

Constitutive expression of several defence-related genes in thenbl3 mutant and wild type. Pathogenesis-related and defence-related genes in the wild type (WT) and nbl3 were analysed using realtime quantitative polymerase chain reaction (RT-qPCR) at the four-leaf stage (before appearance of visible lesions in $n b l 3$ ). The results were obtained using three independent biological replicates. Pathogenesisrelated genes include OsPR1b(LOC_Os07g03710 ), OsPR2 (LOC_Os01g71340 ),OsPR3 (LOC_Os10g39680 ), OsPR5(LOC_Os12g43380 ), OsPR8(LOC_Os10g28080 ), and OsPR10 (LOC_Os12g36880 ). Defence-

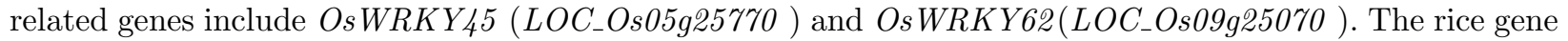
OsActin (LOC_Os03g50885) was used as an internal control. Data are shown as means $\pm \mathrm{SD}, \mathrm{n}=3$ (** $P$ $<0.01$; Student's $t$ test).

\section{FIGURE 3}

Cloning of $O s N B L 3$ and its expression patterns. (a) A schematic diagram showing the T-DNA insertion event in the nbl3mutant. The triangle indicates the T-DNA insertion site in thenbl3 mutant. 
Black boxes indicate exons, lines indicate introns, and white boxes indicate the untranslated regions. The primers used to confirm the insertion event and conduct expression analysis are marked. (b) Confirmation of the T-DNA insertion event by PCR using the specific primers (L3/JD-F, R3/JD-R and JD-F/JD-R); HH and Hh indicate homozygous and heterozygous mutant plants, respectively. (c) and (d) Expression analysis of OsNBL3 in the wild-type (WT) and nbl3 plants by semi-quantitative real-time (RT-PCR) and realtime quantitative PCR, (RT-qPCR), respectively. The rice gene Actin1 (LOC_Os07g38730 ) was used as an internal control, data are shown as means $\pm \mathrm{SD}, \mathrm{n}=3\left({ }^{* *} P<0.01\right.$; Student's $t$ test). (e) RT-qPCR analysis of OsNBL3 expression patterns in different tissues of wild-type plants. Flag leaf (L1), second leaf (L2), third leaf (L3), fourth leaf (L4), sheath (Sh), stem node 1 (Sn1), stem node 2 (Sn2), basal node (Bn), panicle $(\mathrm{Pa})$, and root $(\mathrm{Ro})$. Data are shown as means $\pm \mathrm{SD}, \mathrm{n}=3$. The rice OsActin (LOC_Os03g50885) gene was used as an internal control. (f) RT-qPCR analysis of the OsNBL3 expression level at different times after inoculation with the Magnaporthe oryzae isolate H535. Two-week-old seedlings were used for inoculation. The seedlings that were sprayed only with $0.025 \%$ Tween 20 were used as a negative control (Mock). The rice OsActin (LOC_Os03g50885) gene was used as an internal control. Data are shown as means $\pm \mathrm{SD}, \mathrm{n}$ $=3\left({ }^{* *} P<0.01 ;\right.$ Student's $t$ test $)$.

\section{FIGURE 4}

RNA interference (RNAi) plants exhibit enhanced disease resistance. (a) Schematic diagrams of the OsNBL3 overexpression (OE) and RNAi structures. Ubi, maize ubiquitin promoter and 3xFlag, three tandem repeat Flag tag. A cDNA fragment from $238 \mathrm{bp}$ to $797 \mathrm{bp}$ downstream of the OsNBL3 ATG was used for the sense and antisense sequence. (b) Leaves of the wild type (WT) and nbl3 were punch inoculated with Magnaporthe oryzae isolate H535 and photographed at 96 hours post inoculation. (c) Expression analysis of OsNBL3in the wild type (WT) and OsNBL3-OE or OsNBL3-RNAi lines by real-time quantitative polymerase chain reaction (RT-qPCR). Data are shown as means $\pm \mathrm{SD}, \mathrm{n}=3(* * P<0.01$; Student's $t$ test). OsNBL3-RNAi lines include R6, R10 and R20, and OsNBL3-OE lines include OE-16, OE21 and OE-23. (d) Lesion length of leaves of the wild-type, nbl3, OsNBL3-OE and OsNBL3-RNAi lines at 96 hours post inoculation with Magnaporthe oryzae isolate H535. (**P<0.01; Student's $t$ test). Data are shown as means $\pm \mathrm{SD}, \mathrm{n}=10$.

\section{FIGURE 5}

Disruption of $O s N B L 3$ leads to enhanced salt tolerance.(a) and (b) Effects of salt treatment on root growth of the wild type and the nbl3 mutant. Fifteen germinated seeds were planted on agar medium containing $100 \mathrm{mM} \mathrm{NaCl}$ for 5 days (b). Those growing on agar medium were used as controls (a). (c) The inhibition rate of root growth of the wild type and the nbl3 mutant by treatment with $100 \mathrm{mM} \mathrm{NaCl}$. Root lengths were measured at different time points. Data are shown as means $\pm \mathrm{SD}, \mathrm{n}=15{ }^{* *} P<$ 0.01; Student'st test). (d) and (e) Photographs of 50-day-old seedlings of the wild type and nbl3 , that were continuously irrigated with $200 \mathrm{mM} \mathrm{NaCl}$ or water only, for twenty days. (f) Real-time quantitative polymerase chain reaction (RT-qPCR) analysis of temporal expression patterns of $O s N B L 3$ in the wild-type seedlings that were continuously irrigated with $200 \mathrm{mM} \mathrm{NaCl}$. The OsActin gene (LOC_Os03g50885) was used as an internal control. Data are shown as means $\pm \mathrm{SD}, \mathrm{n}=3\left({ }^{* *} P<0.01\right.$; Student's $t$ test). (g) RT-qPCR analyses of constitutive expression of several salt tolerance-related genes in $n b l 3$ and wild-type seedlings. TheOsActin gene (LOC_Os03g50885) was used as an internal control. Data are shown as means $\pm \mathrm{SD}, \mathrm{n}=3\left({ }^{* *} P<0.01 ;\right.$ Student's $t$ test $)$.

\section{FIGURE 6}

OsNBL3 is a mitochondria-localized P-type pentatricopeptide repeat (PPR) protein. (a) Schematic diagrams of the structures of OsNBL3 and 3N-GFP, in which the N terminus of OsNBL3 was fused in frame with green fluorescent protein (GFP). The mitochondrion signal peptide (SP) and PPR repeats (P) predicted by https://ppr.plantenergy.uwa.edu.au/ are indicated. (b) Confocal microscopic images showing co-localization of 3N-GFP and pCXSN-COX4-RFP, in which the Mito marker COX4 was fused with the red fluorescent protein (RFP). Bar $=25 \mu \mathrm{m}$. (c) Confocal microscopic images showing co-localization of 
$3 \mathrm{~N}-\mathrm{GFP}$ and a Mito tracker in rice protoplasts. Bar $=20 \mu \mathrm{m}$.

\section{FIGURE 7}

OsNBL3 participates in the splicing of nad5 intron 4.(a) Semi-quantitative real-time (RT)-PCR analyses of all rice mitochondria intron-containing genes and other mitochondrial genes in the wild type and nbl3 mutant. U and S indicate unspliced and spliced transcripts, respectively. (b) Real-time quantitative PCR (RT-qPCR) analyses of all 23 group II introns in mitochondria. Histogram showing the log2 ratio of spliced to unspliced RNA in nbl3compared with the corresponding value for the wild type. The results are from three biological replicates, data are shown as means \pm SD and $n=3$. (c) Schematic representation of the rice mitochondrial nad5gene. Black boxes indicate exons and black curved lines indicate introns. (d) Semi-quantitative RT-PCR analysis of four nad5intron splicing events in the wild type and nbl3 mutant.

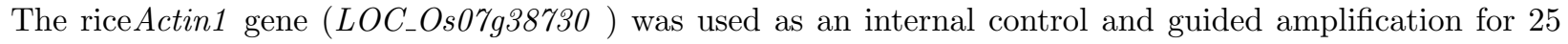
cycles.

\section{FIGURE 8}

$O s N B L 3$ mutation affects mitochondrial morphology and elevates use of alternative respiratory pathways. (a) Transmission electron microscope images of mitochondria in wild-type (WT) and $n b l 3$ leaves. The red arrows indicate mitochondria $(\mathrm{Mt})$. Bar $=0.5 \mu \mathrm{m}$. (b) Real-time quantitative polymerase chain reaction (RT-qPCR) analyses of alternative oxidase (AOX) genes and NADH dehydrogenase (ND) genes. The $\operatorname{rrn} 18$ (LOC_Os03g42530) gene was used as an internal control. Data are shown as means $\pm \mathrm{SD}, \mathrm{n}=$ 3 (** $P<0.01$; Student's $t$ test).

\section{Hosted file}

FIGURES.pdf available at https://authorea.com/users/368840/articles/487848-osnbl3-amitochondria-localized-pentatricopeptide-repeat-protein-is-involved-in-splicing-nad5intron-4-and-responding-to-biotic-and-abiotic-stresses 